\title{
Application of extracorporeal membrane oxygenation for diffuse alveolar hemorrhage due to Wegener's Granulomatosis
}

\author{
Özgür Altınbaş ${ }^{1}$, Funda Gök², Mehmet Işık ${ }^{3}$, Ömer Tanyeli ${ }^{3}$, Yüksel Dereli ${ }^{3}$, Niyazi Görmüşs
}

1) Training and Research Hospital, Department of Cardiovascular Surgery, Konya, Turkey
${ }^{2)}$ Necmettin Erbakan University, Meram Medical Faculty, Department of Anesthesiology and Intensive Care, Konya, Turkey
${ }^{3)}$ Necmettin Erbakan University, Meram Medical Faculty, Department of Cardiovascular Surgery, Konya, Turkey

\begin{abstract}
Objective: Wegener's granulomatosis is a systemic vasculitis, characterized by multifocal vascular necrotizing inflammation and granulomatosis that affects small and medium-sized blood vessels. It is associated with antineutrophil cytoplasmic autobody and causes alveolar hemorrhage and renal insufficiency leads to high mortality rates. Extracorporeal membrane oxygenation (ECMO) is a supportive treatment choice for patients with severe respiratory failure. We present an ECMO application to 64 year-old female patient with acute alveolar hemorrhage and respiratory failure due to Wegener's granulomatosis under CPR.

Case Report: A 64 year-old female patient, previously diagnosed with chronic renal failure due to Wegener's granulomatosis, admitted to our hospital for routine hemodialysis programme. At the end of the first hour of hemodialysis a worsening in her general condition occured and she required intubation and mechanical ventilation. Computerized thorax tomography revealed bilateral intraalveolar hemorrhage. Although proper medical therapy the patient had respiratory arrest. Despite successful CPR, adequate oxygenation couldn't be reached, and ECMO treatment was initated. Clinical, radiological and laboratory findings showed improvement two days after ECMO initation but the patient died due to multiorgan failure in the fifth day of the therapy.

Conclusion: Diffuse alveolar hemorrhage is a life-threatining complication associated with Wegener's granulomatosis. Mechanical ventilator dependent respiratory failure may develop and this process can be mortal. ECMO is a reasonable management option for such clinical situations.
\end{abstract}

Keywords: Wegener's granulomatosis, alveolar hemorrhage, xtracorporeal membrane oxygenation, respiratory failure. 


\section{Introduction}

Wegener's granulomatosis is one of the ANCA-associated vasculitis characterized by multifocal vascular necrotizing inflammation and granulomas that commonly present with a pulmonary-renal syndrome. ${ }^{(1)} \mathrm{Al}-$ veolar hemorrhage and concomitant glomerulonephritis causing renal insufficiency are associated with high mortality. ${ }^{(2)}$ In these clinical situations ECMO, which is a mode of extracorporeal life support that augments oxygenation, ventilation and/or cardiac output, can be performed. ${ }^{(3)}$

In this study we report an ECMO application to 64 year-old female patient previously diagnosed with Wegener's granulomatosis with acute alveolar hemorrhage and respiratory failure.

\section{Case Report}

A 64 year-old female patient, previously diagnosed with chronic renal failure due to Wegener's granulomatosis, admitted to our hospital for routine hemodialysis programme with systemic anticoagulant. At the end of the first hour of hemodialysis therapy, haemoptysis developed in the patient. Her general condition continued to deteriorate and required intubation and mechanical ventilation. Computerized thorax tomography revealed bilateral intraalveolar hemorrhage (Fig-1).

Figure 1. Intra alveolar hemorrhage focuses in thorax CT image

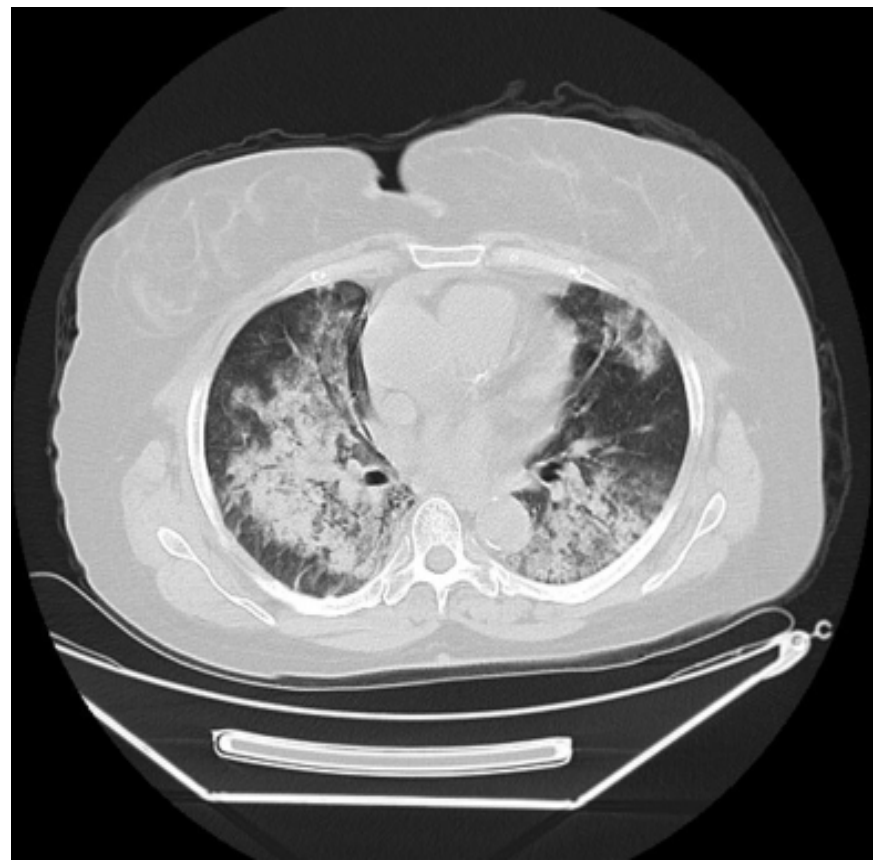

Although proper medical therapy the patient had respiratory arrest. The patient gave response to the CPR after 20 minutes, but adequate oxygenation could not be reached, pO2 was $42 \mathrm{mmHg}$ and $\mathrm{pCO} 2$ was 75 $\mathrm{mmHg}$ thus, veno-venous (right subclavian vein-right femoral vein) ECMO treatment was initated at the bedside of the intensive care unit.

Continue veno-venous hemodiafiltration was started as an additional therapy. Her oxygenation dramatically ameliorated after the begining of this procedure. $\mathrm{pO} 2$ levels increased to $76 \mathrm{mmHg}$ and $\mathrm{pCO} 2$ levels declined to $41 \mathrm{mmHg}$. Haemopthysis decreased; clinical, radiological and laboratory findings showed improvement two days after ECMO initation.

The ECMO lines and oxygenator were not covered. During the ECMO therapy, systemic anticoagulation performed with standart heparine to prevent coagulation in the system of ECMO. Systemic anticoagulation was started just before ECMO set up. APTT levels was tried to kept around 80-100 seconds, thus two-hour APTT controls were performed. There was no serious worsening in clinical situation due to heparine but hemoglobin and hematocrit levels decreased and leaking bleeding at the entrance of the cannula was seen. This clinical situation was achieved with blood transfusion. There was no thrombus or failure in oxygenator in following days.

In the fourth day of the therapy clinical situation got worsening due to multiorgan failure. Although appropriate therapy including liver and kidney protective agents and antibiotics, patient died in the fifth day of ECMO therapy.

\section{Conclusion}

A systemic necrotizing vasculitis called granulomatosis with polyangiitis (Wegener's granulomatosis), affects small and medium sized blood vessels. The main clinical characteristics involve the upper and/or lower respiratory tract and kidneys. ${ }^{(4)}$

Although rare, because of high mortality rates, diffuse alveolar hemorrhage is an important complication. (5) Mortality rates may up to $80 \%$ when the $\mathrm{PaO} 2 / \mathrm{FiO} 2$ ratio under $100 \mathrm{mmHg}$ in mechanically ventilated patients. ${ }^{(6)}$

The most of the patients with diffuse alveolar hem- 
orrhage present with haemoptysis and may have a hypoxemic respiratory failure, although appropriate positioning, intubation and mechanic ventialtion. ECMO application as a rescue thearpy in patients with severe respiratory failure has shown to improve outcomes. ${ }^{(7)}$

ECMO can be used as an advanced circulatory and vantilatory support system in patients with refractory hypoxaemia and/or cardiac failure when the conventional treatment fails. ${ }^{(8)}$

In a study Schmidt et al declared that veno-arterial ECMO is useful in cardiac or cardiorespiratory fail-

\section{References}

1. Savran Y, Gencpinar T, Aydın K, Eroz E. Concurrent Extracorporeal Membrane Oxygenation, Plasmapheresis and Continuous Renal Replacement Therapy in a Case of Wegener's Granulomatosis. Annals of International Medical and Dental Research. 2016; 2(5): 1-3

2. Kallenberg CG. Pathophysiology of ANCA-associated small vessel vasculitis. Curr Rheumatol Rep. 2010; 12(6): 399-405

3. Mosier JM, Kelsey M, Raz Y, Gunnerson KJ, Meyer R, Hypes CD et al.Extracorporeal membrane oxygenation (ECMO) for critically ill adults in the emergency department: history, current applications, and future directions. Critical Care. 2015; 19: 431-438

4. Comarmond C, Cacoub P. Granulomatosis with polyangiitis (Wegener): clinical aspects and treatment. Autoimmun Rev. 2014; 13(11): 1121-5

5. Guo Z, Li X, Jiang LY, Xu LF. Extracorporeal Membrane Oxygenation fort he Management of Respiratory Failure Caused by Diffuse Alveolar Hemorrhage. J Extra Corpor Technol. 2009; 41(1): 37-40 ure, while veno-venous is useful in respiratory failure without cardiac compromise. Additionally, respiratory ECMO can be used as a bridge to recovery to gain time for the treatment of underlying disease. ${ }^{(9)}$ In conclusion, diffuse alveolar hemorrhage can be life-threatining clinical situations of the various vasculitis syndromes. ECMO is a reasonable management option for patients with diffuse alveolar hemorrhage associated severe, refractory hypoxemic respiratory failure as a respiratory support to maintain life and supportive therapy providing time for diagnosis and treatment of the underlying causative disease until its control or resolution.

6. Vasilyev S, Schaap RN, Mortensen JD. Hospital survival rates of patients with acute respiratory failure in modern respiratory intensive care units. An international, multicenter,prospective survey. Chest. 1995; 107: $1083-8$

7. Peek GJ, Mugford M, Tiruvoipati R et al. CESAR trial collaboration efficacy and economic assessment of conventional ventilatory support versus extracorporeal membrane oxygenation for severe adult respiratory failure: a multicenter randomized controlled trial. Lancet. 2009 ; 374(9698): 1351-63

8. Rawal G, Kumar R, Yadav S. ECMO Rescue Therapy in Diffuse Alveolar Haemorrhage: A Case Report with Review of Literature. J Clin Diagn Res. 2016; 10(6): 1-4

9. Schmidt WH, Petermann A, Visouli A, Zarogoulidis P, Darwiche K, Kougioumtzi I et al. Successful application of extracorporeal membrane oxygenation due to pulmonary hemorrhage secondary to granulomatosis with polyangiitis. Drug Des Ther. 2013; 7: 627-633

Received: 08/05/2018

Accepted: 22/08/2018

Published: 15/09/2018

Disclosure and conflicts of interest:

The authors declare no conflict of interest.

Corresponding author:

Özgür Altınbaş

Mail: ozgur_altinbas@yahoo.com 